\title{
Naringin protects against non-alcoholic fatty liver disease by promoting autophagic flux and lipophagy
}

\author{
Lingling Guan ${ }^{1}$, Lan Guo ${ }^{1}$, Heng Zhang ${ }^{2}$, Hao Liu², Yuan Qiao², Wenling Zhou ${ }^{2}$, \\ Yuanyuan Zhai ${ }^{2}$, Xu Yan ${ }^{2}$, Daichuan Cheng ${ }^{1}$, Xiuli Men ${ }^{1}$, and Liang Peng ${ }^{2}$ \\ ${ }^{1}$ North China University of Science and Technology \\ ${ }^{2}$ China Japan Friendship Institute of Clinical Medicine Research
}

October 20, 2021

\begin{abstract}
Abstract Background and Purpose: The autophagic degradation of lipid droplets (LDs), termed lipophagy, is the main mechanism contributing to lipid consumption in hepatocytes. The identification of effective and safe natural compounds that target lipophagy to eliminate excess lipids may be a potential therapeutic strategy for non-alcoholic fatty liver disease (NAFLD). Here, we investigated the effects of naringin on NAFLD and the underlying mechanism. Experimental Approach: The role of naringin was investigated in mice fed a high-fat diet (HFD) to induce NAFLD, as well as in AML12 cells and primary hepatocytes stimulated by palmitate (PA). Transcription factor EB (TFEB)-knockdown AML12 cells and hepatocyte-specific TFEB-knockout mice were also used for the mechanism study. In vivo and in vitro studies were conducted using transmission electron microscopy, immunofluorescence techniques and western blot analysis. Key Results: We found that naringin treatment effectively relieved HFD-induced hepatic steatosis in mice and inhibited palmitate (PA)-induced lipid accumulation in hepatocytes. The increased p62 and LC3-II levels observed with excess lipid-support autophagosome accumulation and impaired autophagic flux. Treatment with naringin restored TFEB-mediated lysosomal biogenesis, thereby promoting the fusion of autophagosomes and lysosomes, restoring impaired autophagic flux and further inducing lipophagy. However, the knockdown of TFEB in hepatocytes or the hepatocyte-specific knockout of TFEB in mice abrogated naringin-induced lipophagy, which eliminated the therapeutic effect of naringin on hepatic steatosis. Conclusion and Implications: These results demonstrate that TFEB-mediated lysosomal biogenesis and subsequent lipophagy play essential roles in the ability of naringin to mitigate hepatic steatosis and suggest that naringin is a promising drug for treating or relieving NAFLD.
\end{abstract}

Naringin protects against non-alcoholic fatty liver disease by promoting autophagic flux and lipophagy

Lingling Guan ${ }^{1,2}$, Lan Guo ${ }^{1}$, Heng Zhang ${ }^{1,2}$, Hao Liu ${ }^{2}$, Yuan Qiao $^{2}$, Wenling Zhou ${ }^{2}$, Yuanyuan Zhai ${ }^{2}$, Xu Yan $^{2}$, Daichuan Cheng ${ }^{1,2}$, Xiuli Men ${ }^{1, \#}$ and Liang Peng ${ }^{2, \#}$

${ }^{1}$ School of Basic Medical Sciences, North China University of Science and Technology, Tangshan, China

2 Beijing Key Laboratory for Immune-Mediated Inflammatory Diseases, Institute of Medical Science, ChinaJapan Friendship Hospital, Beijing, China

\section{\# Correspondence}

Xiuli Men, School of Basic Medical Sciences, North China University of Science and Technology, Tangshan 063210, China. E-mail: xiulimen@126.com

Liang Peng, Beijing Key Laboratory for Immune-Mediated Inflammatory Diseases, Institute of Medical Science, China-Japan Friendship Hospital, Beijing 100029, China. E-mail:pengliang8028@163.com 


\title{
Acknowledgements
}

This work was supported by grants from National Nature Science Foundation of China (81970713 and 82170817), Beijing Municipal Natural Science Foundation of China (7182147), Capital's Funds for Health Improvement and Research (2018-2-4062), and Joint Project of BRC-BC (Biomedical Translational Engineering Research Center of BUCT-CJFH, Grant No. XK2020-10).

\section{Author contributions}

XLM, LP and LLG jointly conceived and designed the whole experiment. LG and HZ helped collect samples and participated in cell culture. HL, YQ and LWZ designed the mice experiment. YYZ, XY and CDC analyzed and interpreted data. XLM, LP and LLG discussed the results and revised the manuscript.

\section{Conflict of interest}

The authors have no conflicts of interest.

\section{Declaration of transparency and scientific rigour}

This Declaration acknowledges that this paper adheres to the principles for transparent reporting and scientific rigour of preclinical research as stated in the BJP guidelines for Design \& Analysis, Immunoblotting and Immunochemistry, Natural Products Research and Animal Experimentation and as recommended by funding agencies, publishers and other organizations engaged with supporting research.

Running title:Naringin mitigates fatty liver by lipophagy

\begin{abstract}
Background and Purpose :The autophagic degradation of lipid droplets (LDs), termed lipophagy, is the main mechanism contributing to lipid consumption in hepatocytes. The identification of effective and safe natural compounds that target lipophagy to eliminate excess lipids may be a potential therapeutic strategy for non-alcoholic fatty liver disease (NAFLD). Here, we investigated the effects of naringin on NAFLD and the underlying mechanism.
\end{abstract}

Experimental Approach :The role of naringin was investigated in mice fed a high-fat diet (HFD) to induce NAFLD, as well as in AML12 cells and primary hepatocytes stimulated by palmitate (PA). Transcription factor EB (TFEB)-knockdown AML12 cells and hepatocyte-specific TFEB-knockout mice were also used for the mechanism study. In vivo and in vitrostudies were conducted using transmission electron microscopy, immunofluorescence techniques and western blot analysis.

Key Results: We found that naringin treatment effectively relieved HFD-induced hepatic steatosis in mice and inhibited palmitate (PA)-induced lipid accumulation in hepatocytes. The increased p62 and LC3II levels observed with excess lipid-support autophagosome accumulation and impaired autophagic flux. Treatment with naringin restored TFEB-mediated lysosomal biogenesis, thereby promoting the fusion of autophagosomes and lysosomes, restoring impaired autophagic flux and further inducing lipophagy. However, the knockdown of TFEB in hepatocytes or the hepatocyte-specific knockout of TFEB in mice abrogated naringin-induced lipophagy, which eliminated the therapeutic effect of naringin on hepatic steatosis.

Conclusion and Implications: These results demonstrate that TFEB-mediated lysosomal biogenesis and subsequent lipophagy play essential roles in the ability of naringin to mitigate hepatic steatosis and suggest that naringin is a promising drug for treating or relieving NAFLD.

\section{What is already known}

Naringin, an active flavanone glycoside in citrus herbs and grapefruit, can exert hepatoprotective and hypolipidaemic effects.

\section{What this study adds}


Naringin promotes TFEB-mediated lysosomal biogenesis and lipophagy to alleviate NAFLD in HFD mice.

\section{What is the clinical significance}

Naringin may be a novel therapeutic drug for the treatment of NAFLD.

TFEB-mediated lysosomal biogenesis is a promising therapeutic target in NAFLD.

\section{Abbreviations}

LDs, lipid droplets; PA, palmitate; AML12, alpha mouse liver-12; DMEM, Dulbecco's modified Eagle's medium; ALT, serum alanine aminotransferase; AST, aspartic acid transaminase; BSA, bovine serum albumin; CQ, chloroquine; DAPI, 4',6-diamidino-2-phenylindole; FBS, fetal bovine serum; H\&E, hematoxylin and eosin; ND, normal diet; HFD, high-fat diet; GTT, glucose tolerance test; TFEB, transcription factor EB; ITT, insulin tolerance test; HDL-C, high-density lipoprotein-cholesterol; LDL-C, low-density lipoproteincholesterol; LAMP1, lysosome-associated membrane protein 1; NAFLD, non-alcoholic fatty liver disease; xsiRNA, small interfering RNA; S6K1, ribosomal protein S6 kinase $\beta-1$; TC, total cholesterol; TG, triglyceride; TEM, transmission electron microscopy

\section{Keywords:}

Non-alcoholic fatty liver disease; Naringin; Lipophagy; TFEB; Autophagic flux

\section{Introduction}

Non-alcoholic fatty liver disease (NAFLD), which is the excessive accumulation of fat in the liver, has a sharply rising incidence parallel with the increasing incidence of obesity and metabolic syndrome worldwide (Lim, Kim \& Targher, 2021). The condition will progress over time, increasing the risk of cirrhosis, endstage liver disease and hepatocellular carcinoma. To date, no surgical procedures or medications have been approved for the effective treatment of NAFLD (Dufour, Caussy \& Loomba, 2020; Ma et al., 2021). In addition, clinically meaningful levels of weight loss have been proven difficult to achieve, especially through sustained lifestyle changes in the form of diet and exercise. Therefore, more studies are urgently needed to develop new drug therapies to effectively treat NAFLD.

Macroautophagy (hereafter called autophagy) is an evolutionarily conserved and lysosome-dependent physiological process that degrades proteins, glycogen and lipids to provide fuel and maintain energy (Morishita \& Mizushima, 2019). Autophagy effectively handles lipid metabolism by selectively degrading lipid droplets (LDs), a process termed lipophagy (Saito et al., 2019; Zhang, Liu, Shen, Tong, Ma \& Lin, 2020). Lipophagy therefore functions to maintain intracellular lipid homeostasis and avoid lipid accumulation (Cui, Sathyanarayan, Lopresti, Aghajan, Chen \& Mashek, 2021). Autophagic flux refers to the dynamic process of autophagy involving the rearrangement of subcellular membranes to sequester cytoplasmic materials, which are delivered to the lysosomes to degrade and recycle (Liao et al., 2021). Appropriate quantities of enzymatically active lysosomes in the acidic cytoplasmic compartment are essential for autophagic flux, especially for autophagosome-lysosome fusion and autolysosome degradation (Mohan, Sorokina, Verdeny, Alvarez \& Lakadamyali, 2019). Previous studies have shown that the biogenesis of lysosomes in mice on a high-fat diet (HFD) is impaired, resulting in inadequate lipophagic activity and excess lipid accumulation. Therefore, promoting lipophagy by inducing lysosomal biogenesis may be a novel therapeutic strategy in NAFLD (Kim et al., 2017b; Lim et al., 2018a).

Transcription factor EB (TFEB) is a key regulator of lysosome biogenesis and autophagy-related gene transcription (Meireles et al., 2018). Normally, TFEB is predominantly diffusely located in the cytoplasm. However, stress conditions, such as nutrient starvation, result in TFEB nuclear translocation, where it binds to the coordinated lysosome expression and regulation elements of target gene promoters to rapidly induce lysosome biogenesis. Recent studies have shown that TFEB overexpression facilitates extensive therapeutic 
effects in HFD or ethanol-induced hepatic steatosis by improving impaired lysosomal biogenesis and enhancing lipolysis (Jeong et al., 2021; Zhang et al., 2020). Therefore, TFEB is a promising therapeutic target of NAFLD by coordinating autophagic induction with lysosomal biogenesis.

Dietary strategies to alleviate NAFLD have been used as an alternative to pharmaceutical interventions in recent years. Naringin is a major and active flavanone glycoside that is ubiquitous in citrus herbs and grapefruit (Alam, Subhan, Rahman, Uddin, Reza \& Sarker, 2014). The broad biological effects of naringin have been well studied for its anti-inflammatory (Akamo et al., 2021), antioxidative (Xi, Fang, Zhao, Jiao \& Zhou, 2014), hypolipidaemic effects (Jung et al., 2003) and antineoplastic activity (Memariani, Abbas, Ul Hassan, Ahmadi \& Chabra, 2020). Moreover, naringin can also ameliorate obesity (Raasmaja et al., 2013), diabetes (Lim et al., 2018b) and atherosclerosis (Wang et al., 2021). These observations emphasize the necessity to evaluate the rational utility of naringin in NAFLD treatment and to investigate the mechanisms involved.

This study therefore explored the possible prevention of NAFLD by naringin in vivo, using the HFD-induced mouse model and in vitro on hepatocytes stimulated by PA. We also performed a mechanistic study with liver-specific TFEB-knockout mice $\left(\mathrm{TFEB}^{\Delta n \varepsilon \pi}\right)$ and littermate controls $\left(\mathrm{TFEB}^{\mathrm{fl} / \mathrm{fl}}\right.$ ). We found that TFEBmediated lysosomal biogenesis was impaired in PA-treated hepatocytes and in the livers of NAFLD mice. Moreover, naringin promoted TFEB-mediated autophagic flux and lipophagy to alleviate hepatic steatosis.

\section{Materials and methods}

\subsection{Animal experiments}

Male C57BL/6J mice were obtained from HFK Bioscience Co., Ltd. (Beijing, China) and weighed between 22 and $25 \mathrm{~g}$ (6-8 weeks old). After acclimatisation for 1 week, mice were stochastically divided into six groups (each group: $\mathrm{n}=6$ ): the normal diet (ND) group; ND with naringin $200 \mathrm{mgkg}^{-1} \mathrm{day}^{-1}$ group; HFD group; HFD with naringin $50 \mathrm{mgkg}^{-1}$ day $^{-1}$ group; HFD with naringin $100 \mathrm{mgkg}^{-1}$ day $^{-1}$ group; and HFD with naringin $200 \mathrm{mgkg}^{-1}$ day $^{-1}$ group. Mice were first fed with ND (10\% kcal from fat) or HFD (60\% kcal from fat) for 2 weeks, followed by 14 weeks of naringin or vehicle treatment by daily intragastric gavage (Figure $1 \mathrm{~A})$.

TFEB flox mice $\left(\mathrm{TFEB}^{\mathrm{fl} / \mathrm{fl}}\right.$ ) were purchased from Cyagen Biosciences (Suzhou, China). The liver-specific TFEB-knockout mice $\left(\mathrm{TFEB}^{\Delta \eta \varepsilon \pi}\right)$ were generated by crossing the TFEB ${ }^{\mathrm{fl} / \mathrm{fl}}$ mice with Albumin-cre transgenic mice (Jackson Laboratory) (Figure 7A). The TFEB flox mice were sorted into four groups: the $\mathrm{TFEB}^{\mathrm{fl} / \mathrm{fl}}$ group; $\mathrm{TFEB}^{\mathrm{fl} / \mathrm{fl}}$ with naringin $200 \mathrm{mgkg}^{-1}$ day $^{-1}$ group; TFEB ${ }^{\Delta n \varepsilon \pi}$ group; and TFEB ${ }^{\Delta n \varepsilon \pi}$ with naringin $200 \mathrm{mgkg}^{-1} \mathrm{day}^{-1}$ group $\left(\mathrm{n}=6\right.$ per group). $\mathrm{TFEB}^{\mathrm{fl} / \mathrm{fl}}$ mice or $\mathrm{TFEB}^{\Delta \eta \varepsilon \pi}$ mice were first fed with HFD for 2 weeks, followed by 14 weeks of naringin or vehicle treatment by daily intragastric gavage.

All the mice were maintained under specific pathogen-free conditions, with the relative humidity ranging between $40 \%$ and $60 \%$, the temperature at $23 \pm 2^{\circ} \mathrm{C}$ and a $12: 12 \mathrm{~h}$ light-dark cycle. During the experimental period, body weight and food intake were measured weekly. At the end of the study, mice were fasted overnight and then anesthetised via the intraperitoneal injection of pentobarbital sodium $\left(60 \mathrm{mgkg}^{-1}\right)$ and euthanised for blood and tissue samples. Animal studies are reported in compliance with the ARRIVE guidelines (McGrath \& Lilley, 2015) and with the recommendations made by the British Journal of Pharmacology . All animal procedures were performed in strict accordance with the criteria outlined in the Guide for the Care and Use of Laboratory Animals prepared by the National Academy of Sciences and published by the National Institutes of Health and with approval from the Ethics Committee of the China-Japan Friendship Institute of Clinical Medicine.

\subsection{Cell culture and treatment}

The alpha mouse liver-12 (AML12) cell line was purchased from the American Type Culture Collection (Manassas, VA, USA). As previously described, primary hepatocytes were isolated from the liver of mice 
(6 weeks old) using a standardised two-step collagenase perfusion procedure (Chen et al., 2019). All cells were cultivated in a high-glucose Dulbecco's Modified Eagle Medium with $1 \%$ streptomycin-penicillin and $10 \%$ foetal bovine serum under standard conditions with $5 \% \mathrm{CO}_{2}$ at $37 \mathrm{deg}$. To establish a cellular model of NAFLD, primary hepatocytes and AML12 cells were exposed to $0.3 \mathrm{mM} \mathrm{PA}$ or $1 \%$ bovine serum albumin (BSA), respectively, for $24 \mathrm{~h}$ and then treated with 20,40 and $80 \mu \mathrm{M}$ naringin. mCherry-GFP-LC3 was transfected into AML12 cells for $48 \mathrm{~h}$ to examine autophagic flux by evaluating the loss of green fluorescent protein (GFP) (Wang et al., 2019; Zummo, Cullen, Honkanen-Scott, Shaw, Lovat \& Arden, 2017). For TFEB silencing, TFEB siRNA or scrambled siRNA were transfected into AML12 cells for $48 \mathrm{~h}$ with Lipofectamine RNAiMAX Reagent (Thermo Fisher Scientific, Loughborough, UK). Steatotic hepatocytes were exposed to compound C (CC; 10 muM; S730, Selleck Chemicals, Houston, TX, United States) with naringin $(80 \mu \mathrm{M})$ for $24 \mathrm{~h}$.

\subsection{Histopathological analysis}

The liver tissues were fixed and preserved in $10 \%$ buffered formalin, processed and trimmed, embedded in paraffin, sectioned to a thickness of 4 or 5 microns and stained with haematoxylin and eosin (H\&E) for histopathological analysis. Frozen sections (10 mum thick) were stained for lipid visualisation with Oil red O. Stained sections were observed using light microscopy (Olympus, Japan). Positive signals were analysed and quantified using Image-Pro Plus 6.0 software (Media Cybernetics, Warrendale, PA, USA).

\subsection{Biochemical analysis}

Serum concentrations of triglyceride (TG), low-density lipoprotein cholesterol (LDL-C), alanine aminotransferase (ALT), total cholesterol (TC), high-density lipoprotein cholesterol (HDL-C) and aspartate aminotransferase (AST) were determined using a 3100 biochemical auto-analyser (Hitachi, Tokyo, Japan). The cellular TG and TC contents were analysed with a commercial kit obtained from Jiancheng Technologies Inc. (Nanjing, China). For the intraperitoneal insulin tolerance test (ITT) or glucose tolerance test (GTT), the mice were fasted overnight, and glucose concentrations were measured immediately before and at 0,15 , 30, 60, 90 and 120 minutes after the intraperitoneal injection of glucose ( $2 \mathrm{gkg}^{-1}$ of body weight) or insulin (0.75 unitskg ${ }^{-1}$ of body weight). Blood glucose levels were immediately detected from tail capillary blood with a Contour Glucose Meter (Bayer, Mountain View, CA, USA).

\section{5.}

\section{LysoTracker Red and BODIPY 493/503 staining}

To stain the lipid droplets (LDs), AML12 cells were incubated with BODIPY 493/503 (1 $\left.\mu \mathrm{gmL}^{-1}\right)$ for 20 min at $37^{\circ} \mathrm{C}$ in a darkroom, and nuclei were incubated with $4^{\prime}, 6$-diamidino-2-phenylindole (DAPI) for 5 min. To determine autolysosome and lipid droplet co-localisation, AML12 cells were incubated with $100 \mathrm{nM}$ LysoTracker Red in DMEM for $1 \mathrm{~h}$ at 37degC, and then BODIPY 493/503 and DAPI staining were performed after $4 \%$ formaldehyde cell fixation. Images were captured using a Zeiss LSM 780 confocal microscope (Carl Zeiss SAS, Jena, Germany).

\subsection{Immunofluorescence}

For immunostaining, the cryosections or hepatocytes were washed thrice with phosphate-buffered saline (PBS) and fixed with $4 \%$ paraformaldehyde, followed by blocking with $5 \%$ BSA at room temperature for 2 h. Primary antibodies were added and incubated overnight at $4 \mathrm{deg} \mathrm{C}$, and unbound antibodies were removed via three washes with PBS before incubation with suitable secondary antibodies for $1 \mathrm{~h}$ at room temperature. The specimens were then rinsed with PBS. DAPI was used for staining nuclei. The stained sections and cells were then mounted, covered with glass coverslips to facilitate high-resolution imaging, sealed with nail polish and stored at -20degC. Fluorescent staining was visualised and imaged using a Zeiss LSM 780 confocal microscope (Carl Zeiss SAS, Jena, Germany).

\subsection{Western blot analysis}


For analysis of protein abundance, total proteins were extracted from AML12 cells and frozen liver tissues using radioimmunoprecipitation assay lysis buffer containing a protease inhibitor cocktail. Cytoplasmic and nuclear fractions were separated using a Nuclear/Cytosol Extraction Kit (BioVision, CA, USA). Samples were resolved by $4 \%-12 \%$ sodium dodecyl sulphate-polyacrylamide gel electrophoresis and transferred to a polyvinylidene fluoride membrane. After blocking with 5\% BSA in Tris-buffered saline supplemented with $0.1 \%$ Tween-20, the membrane was incubated overnight at $4 \mathrm{degC}$ with primary antibody. Corresponding secondary antibodies were labelled with horseradish peroxidase (1:2000) and incubated for $2 \mathrm{~h}$ at $37 \mathrm{degC}$. Next, the blots were developed on the film using the Enhanced Chemiluminescent System (ECL, Bio-Ras Laboratories, Hercules, CA, USA). The following antibodies were used: TFEB (1:2000), p62 (1:1500), LC3 (1:2000), ATP6V1A (1:2000), p-S6K1 (1:2000), LAMP1 (1:1000), p-adenosine monophosphate-activated protein kinase (AMPK) (1:2000) and Beclin1 (1:1500).

\subsection{Transmission electron microscopy}

Autophagosome ultrastructure was evaluated by transmission electron microscopy (JEM-1400PLUS, Tokyo, Japan). Liver tissues and hepatocytes were fixed in $2.5 \%$ glutaraldehyde or $2 \%$ paraformaldehyde for $12 \mathrm{~h}$ at $4 \mathrm{deg} \mathrm{C}$, post-fixed in buffered $1 \%$ osmium tetroxide for $1 \mathrm{~h}$ at $4 \mathrm{degC}$, then dehydrated and infiltrated with Spurr's resin. The tissues were then cut using an ultramicrotome (RMC/MTX; Elexience). The obtained ultrathin sections (60-80 nm) were mounted on copper grids and stained with $4 \%$ uranyl acetate and $0.2 \%$ lead citrate.

\subsection{Antibodies and reagents}

Naringin was purchased from MedChemExpress (Monmouth Junction, NJ, USA). It was dissolved in $\mathrm{H}_{2} \mathrm{O}$ for in vitro and in vivo experiments. PA (P9767), fatty acid-free BSA (A7030), Oil Red O (O0625), chloroquine (CQ, C6628), LC3 antibody (L8918) and P62 antibody (P0067) were obtained from Sigma-Aldrich (St. Louis, MO, USA). BODIPY 493/503 (D3922) and DAPI (D1306) were purchased from Thermo Fisher Scientific (Pittsburgh, PA, USA). Mouse antibodies specific for TFEB (13372-1-AP), ATP6V1A (171151-AP) and antibodies against $\beta$-actin (HRP-60008) were obtained from Proteintech (Wuhan, China). AdmCherry-GFP-LC3 (C3011) and LysoTracker Red (C1046) were obtained from Beyotime (Shanghai, China). Antibodies against phosphorylated 5' AMPK (2535S), lysosome-associated membrane protein 1 (LAMP1; 15665) and phosphorylated ribosomal protein S6 kinase $\beta-1$ (p-S6K1; 9205) were purchased from Cell Signaling Technology (Beverly, MA, USA). Antibodies against histone H3 (ab1791) and Beclin1 (ab62557) were obtained from Abcam (Cambridge, MA, USA). Compound C was obtained from Selleck Chemicals (Houston, TX, USA).

\subsection{0.}

\section{Statistical analysis}

The data and statistical analysis comply with the recommendations of the British Journal of Pharmacology on experimental design and analysis in pharmacology (Curtis et al., 2018). The experiments were designed to establish blinding, randomisation and equal size. All group sizes are the number of independent values, and statistical analyses were performed using these independent values. The statistical analyses were performed only for experiments wherein each group was at least $n=5$. The significance of differences was calculated using one-way ANOVA to compare the effect of one variable or two-way ANOVA to compare the effects of two independent variables followed by Tukey's post hoc test using SPSS 19.0 (Chicago, IL, USA). Post hoc tests were run only if $\mathrm{F}$ achieved $\mathrm{P}<0.05$, and no significant variance in homogeneity was observed. When outliers were involved or excluded in the analysis, they were mentioned within the figure legend. The difference was indicated statistically significant when $\mathrm{P}<0.05$. Data were presented as mean $\pm \mathrm{SEM}$. To control for unwanted sources of variation, data were normalised.

\subsection{Nomenclature of targets and ligands}

Key protein targets and ligands in this article are hyperlinked to corresponding entries in 
http://www.guidetopharmacology.org, the common portal for data from the IUPHAR/BPS Guide to PHARMACOLOGY (Harding et al., 2018), and are permanently archived in the Concise Guide to PHARMACOLOGY 2019/20 (Alexander et al., 2019).

\section{Results}

\subsection{Naringin has a therapeutic effect on HFD-induced hepatic steatosis}

We first explored whether naringin has a protective role in HFD-induced hepatic steatosis. A continuous HFD for 16 weeks led to a significant increase in body weight and serum TG, TC, LDL-C and LDL-C/HDL-C levels, which were in turn decreased in the liver by naringin supplementation (Figures 1B-1G). In addition, GTT and ITT assays revealed that naringin lowered blood glucose and improved insulin resistance in HFDfed obese mice (Figures 1H and 1I). These results occurred without affecting the food intake (Figure 1J). Furthermore, the histological analysis of liver tissues revealed more LDs in the liver of HFD-fed mice than in the control group. Conversely, naringin administration decreased the number of LDs in the liver (Figures $2 \mathrm{~A}-$ 2C). Meanwhile, serum AST and ALT levels and liver TG levels were also decreased by naringin treatment in HFD-fed mice (Figures 2D-2F). Collectively, these results demonstrate that naringin could significantly improve systemic glucose metabolism and alleviate HFD-induced hepatic steatosis.

\subsection{Naringininhibited PA-induced lipid accumulation in hepatocytes}

Based on the results of the cell counting kit- 8 assay, a hepatic in vitro model of cellular steatosis was established in AML12 cells and primary mouse hepatocytes by stimulating the cells with $0.3 \mathrm{mM}$ PA. Naringin concentrations of 20, 40 and $80 \mu \mathrm{M}$ were chosen for the subsequent in vitro experiments (Figures $3 \mathrm{~A}-3 \mathrm{D}$ ). The number of LDs stained by BODIPY 493/503 or Oil red O markedly increased in the PA-treated group, and this increase was significantly decreased by naringin treatment (Figures $3 \mathrm{E}-3 \mathrm{~J}$ ). In addition, naringin also caused a reduction in hepatocellular TG and TC levels in a dose-dependent manner (Figures 3K-3N). These results indicate a role for naringin in inhibiting intracellular lipid accumulation in vitro .

Naringin mitigates hepatic steatosis by restoring autophagic flux

We next examined the effect of lipid challenge on autophagy in cultured hepatocytes and mouse liver tissues. Western blot results revealed that the protein levels of autophagy markers LC3-II and autophagy substrate p62 were significantly increased in steatotic hepatocytes in vitro and in vivo, indicating that hepatic steatosis may increase basal autophagy but impair autolysosome degradation or autophagic flux (Figures 4A and 4B).

To further explore this, we evaluated LC3-II levels, followed by treatment with or without CQ, an inhibitor that blocks the fusion of autophagosomes with lysosomes, to monitor autophagic flux. LC3 net flux was determined by subtracting the densitometry value of normalised LC3-II in non-CQ samples from CQ-treated samples. Higher values represent an increase in LC3 flux. As shown in Figure 4C, hepatic steatosis significantly reduced net LC3-II flux. Consistent with this result, autophagic flux impairment in PA-treated hepatocytes was confirmed by mCherry-GFP-LC3 analysis, a more robust method of detecting autophagic flux. Autophagosomes show dual GFP and mCherry fluorescence, whereas autolysosomes mainly exhibit red fluorescence since GFP fluorescence is quenched in the acidic intralysosomal environment. Therefore, the decreased GFP fluorescence relative to the mCherry fluorescence indicates increased autophagic flux. As expected, GFP fluorescence was enhanced in PA-treated AML12 cells. The above results demonstrate that autophagic flux is obviously blocked in steatotic hepatocytes (Figure 4D).

We further evaluated the role of naringin in autophagic flux and found naringin treatment significantly promoted autophagy induction and autolysosome degradation (i.e., autophagic flux), as evidenced by increased LC3-II protein level and decreased p62 protein level in the liver of HFD-fed mice and PA-treated hepatocytes (Figures 4A and 4B). Moreover, net LC3 flux and mCherry-GFP-LC3 analyses further verified that naringin can restore impaired autophagic flux (Figures 4C and 4D). Of note, transmission electron microscopy results 
further confirmed that naringin significantly increased the number of autophagosomes and lysosomes but decreased the number of LDs in PA-treated hepatocytes (Figure 4E). Therefore, we speculated that naringin may have therapeutic potential in NAFLD by promoting lysosome biogenesis to activate autophagic flux and lipophagy. Further assessment using CQ indicated that pharmacological inhibition of autophagic flux abolished the protective effects of naringin on lipid deposition (Figure $4 \mathrm{~F}$ ). Taken together, these results suggest that naringin alleviates hepatic steatosis by restoring autophagic flux and lipophagy.

\subsection{Naringinrestores autophagic fluxby stimulating lysosome biogenesis}

To determine whether the alterations in autophagic flux could be explained by changes in lysosomal biogenesis, we evaluated the regulation of TFEB. TFEB regulates the expression of lysosome-related genes by translocating into the nucleus, serving as a master regulator for lysosomal biogenesis (Wang et al., 2017b). Western blotting assays revealed naringin treatment promoted TFEB nuclear translocation and its downstream lysosome-related genes, such as ATP6V1A and LAMP1, in the PA-treated AML12 cells and liver tissues of HFD-fed mice (Figures 5A-5E). Increased TFEB nuclear translocation in naringin-treated cells was further verified by confocal microscopy analysis (Figure $5 \mathrm{~F}$ ).

To directly analyse autophagosome-lysosome fusion, we evaluated co-localisation between the autophagosomal marker LC3 and lysosomal marker LAMP1. Naringin remarkably increased the number of lysosomes and autolysosomes in steatotic AML 12 cells (Figure 5G). Next, lysosomes were visualised with LysoTracker Red, and LDs were stained with BODIPY 493/503. Immunofluorescence data showed that naringin restored lipophagy in steatotic AML12 cells, as evidenced by increased co-localisation of LysoTracker Red and BODIPY 493/503 (Figure 5H). Overall, these findings suggest that naringin promoted autophagic flux and alleviated lipid accumulation, possibly due to increased lysosomal biogenesis.

\subsection{Inhibition of AMPK/TFEB pathway attenuatednaringin-induced lysosomal biogenesis and} lipophagy in hepatocytes

We next carried out a transient TFEB knockdown in AML12 cells to verify the effect of TFEB on the regulation of lysosome-related target gene expression and investigated whether TFEB is involved in the beneficial effect of naringin on lysosomal biogenesis and lipophagy. As expected, TFEB downregulation decreased the expressions of LAMP1 and ATP6V1A in AML12 cells (Figures 6A and 6B). Moreover, the knockdown of TFEB largely attenuated the naringin-induced increase in the relative fluorescence signal of mCherry and co-localisation of LAMP1 and LC3, resulting in reduced lipophagy, as evidenced by LysoTracker and BODIPY 493/503 double staining (Figure 6E-G). Thus, these data suggest that naringin reduced lipid accumulation by inducing TFEB-mediated lysosome biogenesis and lipophagy.

Previous studies have linked TFEB nuclear translocation to the AMPK-mTOR signalling pathway (Wang et al., 2019) . To determine whether naringin-induced TFEB nuclear translocation was correlated with the activation of AMPK, we performed western blotting assays on cells exposed to compound C (a wellknown AMPK inhibitor) to evaluate its impact on the phosphorylation levels of AMPK and S6K1, a protein controlling the anabolic arm of the mTORC1 pathway. Remarkably, the inhibition of AMPK abrogated the increase in S6K1 dephosphorylation and AMPK phosphorylation. Beclin 1, a cytoplasmic protein necessary in the formation of autophagosomes, exhibited similar changes as AMPK phosphorylation. AMPK inhibition also attenuated naringin-induced TFEB nuclear translocation (Figures $6 \mathrm{C}$ and $6 \mathrm{D}$ ). Thus, these results indicated that naringin restored lipophagy and reduced hepatic steatosis through the AMPK/TFEB pathway.

\subsection{TFEB-mediated lipophagy is required to promote the beneficial effects of naringin in NAFLDin vivo}

To further determine whether TFEB-mediated lysosomal biogenesis and lipophagy are essential to promoting the beneficial effects of naringin on NAFLD, we used the Cre-lox system to generate hepatocyte-specific TFEB-knockout C57BL6 mice $\left(\mathrm{TFEB}^{\Delta \eta \varepsilon \pi}\right.$ ) by crossing Albumin-cre mice with homozygous TFEB floxed $\left(\mathrm{TFEB}^{\mathrm{fl} / \mathrm{fl}}\right.$ ) mice (Figures 7A and 7B). As expected, hepatocyte-specific TFEB knockout aggravated HFDinduced hepatic steatosis (Figures $7 \mathrm{C}-7 \mathrm{H}$ ) and resulted in a severe autophagic flux blockage, as evidenced by 
the accumulation of p62 and LC3 (Figures 7I-7K and 7M). Moreover, hepatocyte-specific TFEB knockout reversed the amelioration effects of naringin on hepatic steatosis or systemic lipid metabolism in NAFLD mice (Figures $7 \mathrm{C}-7 \mathrm{H}$ ). In addition, the beneficial effects of naringin on autophagic flux (Figures 7I-7K and $7 \mathrm{M}$ ) and lysosomal biogenesis (Figures $7 \mathrm{~L}$ and $7 \mathrm{~N}$ ) were also blocked by TFEB knockout. These discoveries illustrate that the beneficial effects of naringin in NAFLD require TFEB-mediated lysosomal biogenesis and lipophagy.

\section{Discussion}

NAFLD has become the most common chronic liver disease worldwide, but currently, only limited therapies exist (Ray, 2021). Naringin, a natural flavonoid, which is mainly found in various citrus fruits, such as oranges and grapefruits, has been reported to ameliorate dyslipidaemia and NAFLD (Jung et al., 2003; Lim et al., 2018b; Mu et al., 2020). However, the underlying mechanism needs to be further clarified. Our current findings indicate that the regulation of autophagic flux or lipophagy is a critical component in the protective effect of naringin in NAFLD. Our data support a model in which excess lipid accumulation decreased lysosomal biogenesis, leading to impaired autophagic flux and rapid progression of hepatic steatosis. Treatment with naringin restored lysosomal biogenesis, reversing the impairment of autophagic flux and improving NAFLD by activating TFEB.

Autophagy is a conserved lysosomal pathway for the degradation of cytoplasmic components (Morishita \& Mizushima, 2019; Singh et al., 2009). Although autophagy was generally regarded as a non-specific degradation pathway, over the years selective forms of autophagy have been identified. Examples of specific forms of autophagy include the selective elimination of damaged ribosomes (ribophagy), mitochondria (mitophagy), and lipophagy (Levine \& Kroemer, 2019; Wyant et al., 2018). Emerging evidence shows that lipophagy plays a pivotal role in lipid homeostasis. Furthermore, impaired lipophagy may be a contributing factor to the development of excessive hepatic lipid accumulation or steatosis that underlies NAFLD (Singh et al., 2009; Zhang, Liu, Shen, Tong, Ma \& Lin, 2020). Some lipid-lowering agents, such as ezetimibe and fenofibrate have been reported to alleviate NAFLD by promoting lipophagy (Kim et al., 2017a; Yoo, Jeong, Ahn, Chung \& Hwang, 2021). Thus, pharmacological targeting of lipophagy is a promising strategy in the treatment of NAFLD (Galluzzi, Bravo-San Pedro, Levine, Green \& Kroemer, 2017; Lin et al., 2013). Of note, autophagy involves initiation, nucleation, expansion, fusion and degradation (Levine \& Kroemer, 2019). The key step in lipophagy is a complete autophagy-lysosome formation and fusion pathway to generate sufficient autophagic cycles for phagocytosing excess lipids. Previous studies indicated that after long-term HFD feeding in mice, autophagosome biogenesis decreased markedly, resulting in impaired lipophagy (Yang, Li, Fu, Calay \& Hotamisligil, 2010). However, recent studies found that a reduction in autophagic flux caused by the impairment of autophagosome-lysosome fusion led to inadequate lipophagy and intracellular lipid accumulation (Jung et al., 2020). In contrast, lipid accumulation in hepatocytes enhances autophagosome biogenesis. The finding also explains why autophagosome marker LC3-II and autophagy substrate p62, which indirectly reflect the inhibition of autophagic degradation, accumulate in the liver of patients with NAFLD and correlate with disease severity (Martinez-Lopez \& Singh, 2015). In our study, we observed increased LC3-II formation was accompanied by p62 accumulation in the livers of HFD-fed mice and PA-treated hepatocytes compared with controls, indicating impaired autophagic flux. Treatment with naringin resulted in a slight increase in the accumulation of LC3-II and significantly decreased p62 levels in a dose-dependent manner, indicating naringin significantly induced autophagic flux activation in steatotic AML12 cells. Consistent with this result, autophagic flux impairment in PA-treated hepatocytes was also assessed by measuring the LC3 net flux and mCherry-GFP-LC3 analysis. By using CQ, a potent inhibitor of autophagosome-lysosome fusion, the alleviation by naringin of steatosis in AML12 cells is eliminated, which further indicates that naringin could restore lipolytic defects caused by insufficient autophagosome-lysosome fusion. These results reveal that the impairment of autophagic flux in NAFLD might be a strong determining factor in steatosis, suggesting that potential therapeutic strategies aimed to restore autophagic flux might contribute to preventing or attenuating NAFLD progression. In fact, in a recent preclinical research, ikarugamycin, alexidine dihy- 
drochloride and digoxin were selected from a 15,000-chemical library for their ability to improve autophagic flux. These agents improved hepatic steatosis in HFD-fed mice and were associated with the reversal of p62 accumulation in hepatocytes (Wang et al., 2017a). Interestingly, in addition to the change in the number of autophagosomes, there were also significant differences in the number of lysosomes among the groups by transmission electron microscopy, which provided a clue for further mechanism study.

Lysosomes are the main digestive organelles that exist in almost all eukaryotic cells. The role of the lysosome in autophagic degradation is critical, as lysosomes provide the acidic $\mathrm{pH}$ and the hydrolases necessary to degrade the autophagic substrates (Ballabio \& Bonifacino, 2020). Several studies in hepatic steatosis documented a decrement in lysosomal biogenesis that leads to defective autophagic clearance (Chao, Ni \& Ding, 2018; Chao et al., 2018). In line with this, our electron microscopy results showed that the number of lysosomes in fatty liver cells was less than that in the control group. Naringin administration restored lysosomal biogenesis. This process is regulated by TFEB, a gene that is the main regulator of lysosomal biogenesis through the transcriptional conversion of target genes closely related to lysosomal structure and function (Sun et al., 2020; Zhang et al., 2018). Some drugs that target TFEB nuclear translocation to promote autophagy and reduce the phenotype have also been reported, such as trehalose and ezetimibe (Jeong et al., 2021; Kim et al., 2017a), indicating the modulation of TFEB is a promising therapeutic target in NAFLD. In our study, treatment with naringin induced the nuclear translocation of TFEB and upregulated the expression of several lysosomal genes. Moreover, loss-of-function approaches based on knockout mice have been used to explore the role of TFEB in regulating lipid homeostasis (Mansueto et al., 2017; Pastore et al., 2016; Settembre et al., 2013). For instance, TFEB muscle-specific conditional knockout mice showed impaired glucose homeostasis with decreased fatty acid oxidation and oxidative phosphorylation (Mansueto et al., 2017). Likewise, in our current study, mice with hepatocyte-specific knockout of TFEB, as well as hepatocytes with TFEB knockdown, ablated the function of naringin in alleviating lipid accumulation. Collectively, these findings indicate that naringin improves insufficient autophagosome-lysosome fusion by inducing TFEB nuclear translocation to restore lysosomal biogenesis, thereby alleviating hepatic steatosis.

We further investigated the upstream pathways involved in the regulatory effect of naringin on TFEB nuclear translocation. Inhibition of mTOR activity is one of the well-established regulatory mechanisms to induce TFEB nuclear translocation. Previously, AMPK has been reported to inhibit mTOR signalling and promote autophagy in many kinds of cells (Levine \& Kroemer, 2019). Steatotic hepatocytes were incubated with compound $\mathrm{C}$ to assay whether AMPK influences the beneficial effect of naringin. AMPK inhibition decreased the expression of Beclin 1, which is a direct target of AMPK, and also attenuated naringin-induced TFEB nuclear translocation. These results indicated that naringin may restore TFEB-mediated lipophagy through AMPK pathway.

In summary, the present study provides solid evidence supporting the therapeutic effect of naringin on NAFLD. This effect was attributed to TFEB-mediated lysosomal biogenesis and lipophagy. Thus, as our findings indicate naringin may be a promising therapeutic agent in the prevention or treatment of NAFLD (Figure 8).

\section{Figure Legends}

Figure 1. Dietary supplementation of naringin (Nar) alleviates body weight gain and improves systemic glucose metabolism in mice fed an HFD. L, low dose, $50 \mathrm{mgkg}^{-1}$ day $^{-1}$. M, medium dose, $100 \mathrm{mgkg}^{-1} \mathrm{day}^{-1}$. $\mathrm{H}$, high dose, $200 \mathrm{mgkg}^{-1} \mathrm{day}^{-1}$. (A) The experimental flow charts. (B) Bodyweight. (C) Bodyweight gain. Serum levels of (D) TG, (E) TC, (F) LDL-C, (G) LDL-C/HDL-C ratio. (H) GTT was performed at week 14 and area under the curve (AUC) of GTT. (I) ITT was performed at week 15 and AUC of ITT. (J) Food intake. Data shown are individual values with means \pm SEM; $n=6$ in each group. ${ }^{*} \mathrm{p}<0.05$ vs. ND + Vehicle; \#p $<0.05$ vs. HFD + Vehicle. n.s., not significant.

Figure 2. Naringin attenuates hepatic steatosis and liver injury in HFD mice. (A) Liver sections were stained with $\mathrm{H} \& \mathrm{E}$ and Oil red $\mathrm{O}$ from mice fed an ND or HFD followed by treatment with either vehicle or different doses of naringin. Scale bar, $50 \mu \mathrm{M}$. (B-C) Histological scores of liver sections. (D-E) Serum ALT and AST 
levels. (F) TG content in the liver. Data shown are individual values with means \pm SEM; $\mathrm{n}=6$ in each group. ${ }^{*} \mathrm{p}<0.05$ vs. ND + Vehicle; $\# \mathrm{p}<0.05$ vs. HFD + Vehicle.

Figure 3. Naringin mitigates lipid accumulation in palmitate-treated mouse hepatocytes. (A-D) CCK-8 assay. (E) Lipid droplets were stained with Bodipy 493/503. Scale bar, $50 \mu \mathrm{m}$. L, low dose, $20 \mu \mathrm{M}$. M, medium dose, $40 \mu \mathrm{M}$. H, high dose, $80 \mu \mathrm{M}$. (F) Lipid droplets were visualized with Oil red O. Scale bar, 50 $\mu \mathrm{m}$. (G-H) Quantitative results of Bodipy 493/503 staining. (I-J) Quantitative results of Oil red O staining. (K-N) Intracellular TG and TC were quantitatively analyzed. Data shown are individual values with means $\pm \mathrm{SEM} ; \mathrm{n}=6$ in each group. ${ }^{*} \mathrm{p}<0.05$ vs. BSA + Vehicle; $\# \mathrm{p}<0.05$ vs. palmitate $(\mathrm{PA})+$ Vehicle.

Figure 4. Naringin attenuates lipid accumulation in hepatocytes by activating autophagy. (A-B) LC3II and P62 proteins levels in HFD-fed mouse liver and palmitate-induced hepatocytes with naringin (200 mgkg $^{-1}$ day $^{-1}$ or $80 \mu \mathrm{M}$ ) as measured by western blotting. (C) AML12 cells were pretreated with naringin $(80 \mu \mathrm{M}, 24 \mathrm{~h})$ with or without CQ and net LC3-II flux was examined using western blotting analysis. (D) Autophagic flux was analyzed with tandem mCherry-GFP-LC3 in AML12 cells. Scale bar, $50 \mu \mathrm{m}$. (E) The ultramicrotome sections were stained for Electron micrographs analysis of hepatocytes. Insets highlight individual autophagosomes (blue arrowheads), lysosomes (red arrowheads), and lipid droplets (black arrowheads). Scale bar, $1 \mu \mathrm{m}$. (F) Fluorescence microscopy images of hepatocytes were stained with Bodipy493/503 in the presence or absence of CQ for $2 \mathrm{~h}$. Scale bar, $50 \mu \mathrm{m}$. Data shown are individual values with means \pm SEM. $\mathrm{n}=6$ in each group. ${ }^{*} \mathrm{p}<0.05$ vs. BSA + Vehicle or $\mathrm{ND}+$ Vehicle; $\# \mathrm{p}<0.05$ vs. PA + Vehicle or HFD + Vehicle; \&p $<0.05$ vs. PA + Naringin.

Figure 5. Naringin promotes lysosomal biogenesis in PA-induced hepatocytes. (A-B) Western blot assay and relative intensity analysis of hepatic nuclear TFEB and its downstream targets, including LAMP1 and ATP6V1A, in HFD-fed mice with or without naringin $\left(200 \mathrm{mgkg}^{-1}\right.$ day $\left.^{-1}\right)$ treatment. (C) Western blot detection of cytoplasm and nuclear TFEB expression in AML12 cells treated with or without naringin $(80 \mu \mathrm{M})$. Relative expression levels were normalized to $\beta$-actin and Histone 3 levels, respectively. (D) Western blot detection of LAMP1 and ATP6V1A expression in AML12 cells. (E) Relative intensity analysis of TFEB, LAMP1 and ATP6V1A in AML12 cells. (F) Fluorescence microscopy images of percentage of nuclear TFEB in AML12 cells treated with naringin $(80 \mu \mathrm{M})$ for $24 \mathrm{~h}$. Scale bar, $50 \mu \mathrm{m}$. (G) Double immunofluorescence of LC3 (red) and LAMP1 (green) and quantitation analysis of colocalisation. Scale bar, $50 \mu \mathrm{m}$. (D) Representative images for Bodipy 493/503 (green) and LysoTracker Red (red) as counterstaining in the absence or presence of palmitate or Naringin $(80 \mu \mathrm{M})$. Scale bar, $50 \mu \mathrm{m}$. Data shown are individual values with means \pm SEM. $\mathrm{n}=6$ in each group. ${ }^{*} \mathrm{p}<0.05$ vs. BSA + Vehicle or ND + Vehicle; $\# \mathrm{p}<0.05$ vs. PA + Vehicle or HFD + Vehicle.

Figure 6. TFEB knockdown weakened the improvement of lysosome biogenesis and autophagosomelysosome fusion induced by naringin. (A-B) Western blot assay and relative intensity analysis revealed that siRNA targeting TFEB interference reduced the upregulation of protein level in nuclear TFEB and its downstream targets, including LAMP1 and ATP6V1A, induced by naringin $(80 \mu \mathrm{M})$ in PA-stimulated AML12 cells compared with matched controls. (C-D) Western blot assay and relative intensity analysis revealed that AMPK inhibition via Compound C abolished the increased AMPK phosphorylation, S6K1 dephosphorylation and Beclin 1 expression induced by naringin $(80 \mu \mathrm{M})$ in PA-stimulated AML12 cells compared with matched controls. (E) Cells were pre-transfection of mCherry-GFP-LC3 cultured with naringin $(80 \mu \mathrm{M})$, and LC3 puncta were detected with fluorescence microscopy. Scale bar, $50 \mu \mathrm{m}$. (F) Double staining of LC3 (red) and LAMP1 (green) to determine the trafficking of autolysosomes. Scale bar, $50 \mu \mathrm{m} .(\mathrm{G})$ Immunofluorescence for Bodipy 493/503 (green) and LysoTracker Red (red) in hepatocytes treated with or without naringin $(80 \mu \mathrm{M})$. Scale bar, $50 \mu \mathrm{m}$. Data shown are individual values with means \pm SEM. $\mathrm{n}=6$ in each group. ${ }^{*} \mathrm{p}<0.05$ vs. BSA + Vehicle; $\# \mathrm{p}<0.05$ vs. PA + Vehicle; $\& \mathrm{p}<0.05$ vs. PA + Naringin.

Figure 7. TFEB-mediated lipophagy is essential for naringin in improving systemic lipid homeostasis. (A) TFEB floxed mice were crossed with albumin-Cre mice to generate TFEB ${ }^{\Delta n \varepsilon \pi}$ mouse. (B) Western blotting analysis of TFEB protein levels in tissues from TFEB ${ }^{\mathrm{f} / \mathrm{fl}}$ and TFEB ${ }^{\triangle n \varepsilon \pi}$ mice. (C) Body weight gain. Serum levels of (D) TG, (E) ALT. (F) TG content in the liver. (G) Liver sections were stained with H\&E and 
Oil red $\mathrm{O}$ from $\mathrm{TFEB}^{\mathrm{f} / \mathrm{fl}}$ and $\mathrm{TFEB}^{\triangle n \varepsilon \pi}$ mice fed an HFD followed by treatment with either vehicle or naringin $\left(200 \mathrm{mgkg}^{-1}\right.$ day $\left.^{-1}\right)$. Scale bar, $50 \mu \mathrm{M}$. (H) Histological scores of liver sections. (I-J) LC3-II and P62 proteins levels as measured by western blotting and relative intensity analysis. (K) Immunostaining of p62 (red) in myocardium sections. Nuclei were counter-stained with DAPI (blue). Scale bar, $100 \mu \mathrm{m}$. (L) The ultramicrotome sections were stained for Electron micrographs analysis of liver tissue. Insets highlight individual autophagosomes (blue arrowheads) and lysosomes (red arrowheads). LD, lipid droplet. Scale bar, $1 \mu \mathrm{m}$. (M) Quantification of the results in panel K. (N) Quantification of the results in panel N. Data shown are individual values with means \pm SEM. $\mathrm{n}=6$ in each group. $\# \mathrm{p}<0.05$, significantly different as indicated. n.s., not significant.

Figure 8. Schematic diagram of the mechanism of Naringin-mediated alleviation of hepatic steatosis. Naringin eliminates the inhibition of TFEB nuclear translocation caused by HFD or PA exposure, enhances autophagy flux and alleviates hepatic steatosis.

Supplementary figure. 1 The hepatocyte-specific knockout of TFEB in mice aggravates obesity and metabolic disorders. (A) Bodyweight. (B) GTT was performed at week 14 and area under the curve (AUC) of GTT. (C) ITT was performed at week 15 and AUC of ITT. Serum levels of (D) TC, (E) LDL-C, (F) HDL-C, (G) AST. (H) Food intake. Data shown are individual values with means \pm SEM; $\mathrm{n}=6$ in each group. Data shown are individual values with means \pm SEM. $n=6$ in each group. $\# p<0.05$, significantly different as indicated. n.s., not significant.

\section{References}

Akamo A, Rotimi S, Akinloye D, Ugbaja R, Adeleye O, Dosumu O, et al. (2021). Naringin prevents cyclophosphamide-induced hepatotoxicity in rats by attenuating oxidative stress, fibrosis, and inflammation. Food and chemical toxicology : an international journal published for the British Industrial Biological Research Association 153:112266.

Alam M, Subhan N, Rahman M, Uddin S, Reza H, \& Sarker S (2014). Effect of citrus flavonoids, naringin and naringenin, on metabolic syndrome and their mechanisms of action. Advances in nutrition (Bethesda, Md) 5: 404-417.

Alexander S, Kelly E, Mathie A, Peters J, Veale E, Armstrong J, et al. (2019). THE CONCISE GUIDE TO PHARMACOLOGY 2019/20: Introduction and Other Protein Targets. British journal of pharmacology: S1-S20.

Ballabio A, \& Bonifacino J (2020). Lysosomes as dynamic regulators of cell and organismal homeostasis. Nature reviews Molecular cell biology 21: 101-118.

Chao X, Ni H, \& Ding W (2018). Insufficient autophagy: a novel autophagic flux scenario uncovered by impaired liver TFEB-mediated lysosomal biogenesis from chronic alcohol-drinking mice. Autophagy 14: 1646-1648.

Chao X, Wang S, Zhao K, Li Y, Williams J, Li T, et al. (2018). Impaired TFEB-Mediated Lysosome Biogenesis and Autophagy Promote Chronic Ethanol-Induced Liver Injury and Steatosis in Mice. Gastroenterology 155: 865-879.e812.

Chen X, Xue H, Fang W, Chen K, Chen S, Yang W, et al. (2019). Adropin protects against liver injury in nonalcoholic steatohepatitis via the Nrf2 mediated antioxidant capacity. Redox biology 21:101068.

Cui W, Sathyanarayan A, Lopresti M, Aghajan M, Chen C, \& Mashek D (2021). Lipophagy-derived fatty acids undergo extracellular efflux via lysosomal exocytosis. Autophagy 17: 690-705.

Curtis M, Alexander S, Cirino G, Docherty J, George C, Giembycz M, et al. (2018). Experimental design and analysis and their reporting II: updated and simplified guidance for authors and peer reviewers. British 
journal of pharmacology 175: 987-993.

Dufour J, Caussy C, \& Loomba R (2020). Combination therapy for non-alcoholic steatohepatitis: rationale, opportunities and challenges. Gut 69: 1877-1884.

Galluzzi L, Bravo-San Pedro J, Levine B, Green D, \& Kroemer G (2017). Pharmacological modulation of autophagy: therapeutic potential and persisting obstacles. Nature reviews Drug discovery 16:487-511.

Harding S, Sharman J, Faccenda E, Southan C, Pawson A, Ireland S, et al. (2018). The IUPHAR/BPS Guide to PHARMACOLOGY in 2018: updates and expansion to encompass the new guide to IMMUNOPHARMACOLOGY. Nucleic acids research 46: D1091-D1106.

Jeong S, Stitham J, Evans T, Zhang X, Rodriguez-Velez A, Yeh Y, et al. (2021). Trehalose causes low-grade lysosomal stress to activate TFEB and the autophagy-lysosome biogenesis response. Autophagy:1-13.

Jung S, Lee W, Park S, Lee K, Choi Y, Choi S, et al. (2020). Diclofenac impairs autophagic flux via oxidative stress and lysosomal dysfunction: Implications for hepatotoxicity. Redox biology 37:101751.

Jung U, Kim H, Lee J, Lee M, Kim H, Park E, et al. (2003). Naringin supplementation lowers plasma lipids and enhances erythrocyte antioxidant enzyme activities in hypercholesterolemic subjects. Clinical nutrition (Edinburgh, Scotland) 22: 561-568.

Kim S, Kim G, Han D, Lee M, Kim I, Kim B, et al. (2017a). Ezetimibe ameliorates steatohepatitis via AMP activated protein kinase-TFEB-mediated activation of autophagy and NLRP3 inflammasome inhibition. Autophagy 13: 1767-1781.

Kim SH, Kim G, Han DH, Lee M, Kim I, Kim B, et al. (2017b). Ezetimibe ameliorates steatohepatitis via AMP activated protein kinase-TFEB-mediated activation of autophagy and NLRP3 inflammasome inhibition. Autophagy 13: 1767-1781.

Levine B, \& Kroemer G (2019). Biological Functions of Autophagy Genes: A Disease Perspective. Cell 176: $11-42$.

Liao Y, Xu J, Qin B, Shi J, Qin C, Xie F, et al. (2021). Advanced oxidation protein products impair autophagic flux in macrophage by inducing lysosomal dysfunction via activation of PI3K-Akt-mTOR pathway in Crohn's disease. Free radical biology \& medicine.

Lim H, Lim YM, Kim KH, Jeon YE, Park K, Kim J, et al. (2018a). A novel autophagy enhancer as a therapeutic agent against metabolic syndrome and diabetes. Nat Commun 9: 1438.

Lim S, Kim J, \& Targher G (2021). Links between metabolic syndrome and metabolic dysfunction-associated fatty liver disease. Trends in endocrinology and metabolism: TEM.

Lim Y, Kim J, Pan J, Kim J, Park T, Kim Y, et al. (2018b). Naringin Protects Pancreatic $\beta$-Cells Against Oxidative Stress-Induced Apoptosis by Inhibiting Both Intrinsic and Extrinsic Pathways in Insulin-Deficient Diabetic Mice. Molecular nutrition \& food research 62.

Lin C, Zhang H, Li M, Xiong X, Chen X, Chen X, et al. (2013). Pharmacological promotion of autophagy alleviates steatosis and injury in alcoholic and non-alcoholic fatty liver conditions in mice. Journal of hepatology 58: 993-999.

Ma N, Wang Y, Xu S, Ni Q, Zheng Q, Zhu B, et al. (2021). PPDPF alleviates hepatic steatosis through inhibition of mTOR signaling. Nature communications 12: 3059.

Mansueto G, Armani A, Viscomi C, D’Orsi L, De Cegli R, Polishchuk E, et al. (2017). Transcription Factor EB Controls Metabolic Flexibility during Exercise. Cell metabolism 25: 182-196.

Martinez-Lopez N, \& Singh R (2015). Autophagy and Lipid Droplets in the Liver. Annual review of nutrition 35: 215-237. 
McGrath JC, \& Lilley E (2015). Implementing guidelines on reporting research using animals (ARRIVE etc.): new requirements for publication in BJP. Br J Pharmacol 172: 3189-3193.

Meireles A, Shen K, Zoupi L, Iyer H, Bouchard E, Williams A, et al. (2018). The Lysosomal Transcription Factor TFEB Represses Myelination Downstream of the Rag-Ragulator Complex. Developmental cell 47: 319-330.e315.

Memariani Z, Abbas S, Ul Hassan S, Ahmadi A, \& Chabra A (2020). Naringin and naringenin as anticancer agents and adjuvants in cancer combination therapy: Efficacy and molecular mechanisms of action, a comprehensive narrative review. Pharmacological research:105264.

Mohan N, Sorokina E, Verdeny I, Alvarez A, \& Lakadamyali M (2019). Detyrosinated microtubules spatially constrain lysosomes facilitating lysosome-autophagosome fusion. The Journal of cell biology 218:632-643.

Morishita H, \& Mizushima N (2019). Diverse Cellular Roles of Autophagy. Annual review of cell and developmental biology 35: 453-475.

Mu H, Zhou Q, Yang R, Zeng J, Li X, Zhang R, et al. (2020). Naringin Attenuates High Fat Diet Induced Non-alcoholic Fatty Liver Disease and Gut Bacterial Dysbiosis in Mice. Front Microbiol 11: 585066.

Pastore N, Brady O, Diab H, Martina J, Sun L, Huynh T, et al.(2016). TFEB and TFE3 cooperate in the regulation of the innate immune response in activated macrophages. Autophagy 12: 1240-1258.

Raasmaja A, Lecklin A, Li X, Zou J, Zhu G, Laakso I, et al.(2013). A water-alcohol extract of Citrus grandis whole fruits has beneficial metabolic effects in the obese Zucker rats fed with high fat/high cholesterol diet. Food chemistry 138: 1392-1399.

Ray K (2021). Examining the prevalence of NAFLD and NASH in a US cohort. Nature reviews Gastroenterology \& hepatology.

Saito T, Kuma A, Sugiura Y, Ichimura Y, Obata M, Kitamura H, et al. (2019). Autophagy regulates lipid metabolism through selective turnover of NCoR1. Nature communications 10: 1567.

Settembre C, De Cegli R, Mansueto G, Saha P, Vetrini F, Visvikis O, et al. (2013). TFEB controls cellular lipid metabolism through a starvation-induced autoregulatory loop. Nature cell biology 15: 647-658.

Singh R, Kaushik S, Wang Y, Xiang Y, Novak I, Komatsu M, et al.(2009). Autophagy regulates lipid metabolism. Nature 458:1131-1135.

Sun X, Shu Y, Yan P, Huang H, Gao R, Xu M, et al. (2020). Transcriptome profiling analysis reveals that ATP6V0E2 is involved in the lysosomal activation by anlotinib. Cell death \& disease 11: 702.

Wang C, Niederstrasser H, Douglas P, Lin R, Jaramillo J, Li Y, et al. (2017a). Small-molecule TFEB pathway agonists that ameliorate metabolic syndrome in mice and extend C. elegans lifespan. Nature communications 8: 2270.

Wang C, Niederstrasser H, Douglas PM, Lin R, Jaramillo J, Li Y, et al. (2017b). Small-molecule TFEB pathway agonists that ameliorate metabolic syndrome in mice and extend C. elegans lifespan. Nat Commun 8: 2270 .

Wang F, Zhao C, Yang M, Zhang L, Wei R, Meng K, et al. (2021). viaFour Citrus Flavanones Exert Atherosclerosis Alleviation Effects in ApoE Mice Different Metabolic and Signaling Pathways. Journal of agricultural and food chemistry 69: 5226-5237.

Wang Y, Zhao H, Li X, Wang Q, Yan M, Zhang H, et al. (2019). Formononetin alleviates hepatic steatosis by facilitating TFEB-mediated lysosome biogenesis and lipophagy. J Nutr Biochem 73: 108214.

Wyant G, Abu-Remaileh M, Frenkel E, Laqtom N, Dharamdasani V, Lewis C, et al. (2018). NUFIP1 is a ribosome receptor for starvation-induced ribophagy. Science (New York, NY) 360:751-758. 
Xi W, Fang B, Zhao Q, Jiao B, \& Zhou Z (2014). Flavonoid composition and antioxidant activities of Chinese local pummelo (Citrus grandis Osbeck.) varieties. Food chemistry 161: 230-238.

Yang L, Li P, Fu S, Calay E, \& Hotamisligil G (2010). Defective hepatic autophagy in obesity promotes ER stress and causes insulin resistance. Cell metabolism 11: 467-478.

Yoo J, Jeong I, Ahn K, Chung H, \& Hwang Y (2021). Fenofibrate, a PPAR $\alpha$ agonist, reduces hepatic fat accumulation through the upregulation of TFEB-mediated lipophagy. Metabolism: clinical and experimental 120: 154798 .

Zhang J, Wang J, Wong Y, Sun X, Chen Y, Wang L, et al. (2018). Docetaxel enhances lysosomal function through TFEB activation. Cell death \& disease 9: 614.

Zhang T, Liu J, Shen S, Tong Q, Ma X, \& Lin L (2020). SIRT3 promotes lipophagy and chaperon-mediated autophagy to protect hepatocytes against lipotoxicity. Cell death and differentiation 27: 329-344.

Zhang Z, Qian Q, Li M, Shao F, Ding W, Lira V, et al. (2020). The unfolded protein response regulates hepatic autophagy by sXBP1-mediated activation of TFEB. Autophagy: 1-15.

Zummo FP, Cullen KS, Honkanen-Scott M, Shaw JAM, Lovat PE, \& Arden C (2017). Glucagon-Like Peptide 1 Protects Pancreatic beta-Cells From Death by Increasing Autophagic Flux and Restoring Lysosomal Function. Diabetes 66: 1272-1285. 